\title{
Redes personales como factores de riesgo y protección en mujeres privadas de libertad ${ }^{*}$
}

\section{Personal networks as risk factors and protection of women in prison \\ Redes pessoais como fatores de risco e proteção mulheres privadas de sua liberdade}

Fecha de recepción: 2019/08/30 | Fecha de evaluación: 2020/07/0 | Fecha de aprobación: 2020/07/06

Maribel Rivera-López

Doctoranda en Ciencias de la Educación en la Universidad de Granada, España Profesora-investigadora de las áreas de psicología social y de educación sociocultural, Universidad Autónoma de Querétaro, México 0000-000I-6850-5054 maribel.rivera@uaq.mx
Fanny T. Añaños

Doctora en Pedagogía Profesora titular del Departamento de Pedagogía y subdirectora del Instituto Universitario de la Paz y los Conflictos (IPAZ), Universidad de Granada, España. / G.I. HISULA, UPTC, Colombia 0000-000I-75I5-1987 fanntab@ugr.es

Para citar este artículo / To reference this article / Para citar este artigo: Rivera-López, M. \& Añaños F. T. (202I). Redes personales como factores de riesgo y protección en mujeres privadas de libertad. Revista Criminalidad, 63(2), 17-32. https://doi. org//0.4774I/17943108.314

\section{Resumen}

El trabajo analiza las redes personales de las mujeres privadas de libertad, identificando factores de riesgo y protección en tres etapas definidas por el ingreso a prisión y orientadas a la reinserción. Se realizó una investigación documental en bases de datos (WOS, Scopus y Google Académico), empleando Mendeley como gestor. Los resultados muestran que, las redes, antes de prisión están permeadas por la violencia y ausencia de apoyo; durante la prisión se dividen en internasexternas y presentan modificaciones relacionales importantes (ruptura de pareja, distanciamiento o no de los/as hijos/as y apoyo de nuevas personas) $y$; en semilibertad se amplían y mejoran estas, favoreciendo la reinserción social, no obstante, la mayoría regresa al entorno familiar y comunitario inicial enfrentándose a los factores de riesgo asociados.

\section{Palabras clave}

Mujeres privadas de libertad, redes personales, reinserción social, factores de riesgo, factores de protección (fuente: Tesauro Criminológico - Instituto de Investigación Interregional de Crimen y Justicia de las Naciones Unidas - UNICRI).

\section{Abstract}

This paper analyzes the personal networks of women in prison, identifying risk and protection factors in three stages, defined by prison entry, aimed at reintegration. Documentary research was carried out on databases (WOS, Scopus and academic Google), using Mendeley as manager. The results show that, before imprisonment, networks are permeated by violence and lack of support; during prison they are divided into internal-external and present important relational modifications (breakup of a couple, distance or not from children and support of new people) and; in day release they expand and improve these, promoting social reinsertion; nevertheless, most return to the initial family and community environment facing the associated risk factors. 


\section{Keywords:}

Women in prison, interpersonal relations, social reintegration, risk factors, protection factors (source: Criminological Thesaurus - United Nations Interregional Crime and Justice Research Institute - UNICRI).

\section{Resumo}

Este artigo analisa as redes pessoais de mulheres privadas de liberdade identificando fatores de risco e proteção em três etapas e definidos pela entrada na prisão, visando a reintegração. Uma pesquisa documental foi realizada em bancos de dados (WOS, Scopus e Google scholar), utilizando Mendeley como gerente. Os resultados mostram que, as redes, antes da prisão são permeadas pela violência e falta de apoio; durante a prisão são divididas em modificações relacionais internas-externas e apresentam importantes (separação do casal, distanciamento ou não dos filhos e apoio de novas pessoas) e; na semi-liberdade são ampliadas e melhoradas, favorecendo a reintegração social, no entanto, a maioria retorna ao ambiente familiar e comunitário inicial diante dos fatores de risco associados.

\section{Palavras-chave}

Mulheres privadas de liberdade, redes pessoais, reintegração social, fatores de risco, fatores de proteção. (fonte: Thesaurus Criminológico - Instituto Inter-regional de Pesquisa em Crime e Justiça das Nações Unidas - UNICRI).

\section{Introducción}

A lo largo de los últimos veinte años se ha incrementado la investigación sobre las mujeres reclusas en España y en el mundo; no obstante, sigue siendo insuficiente para visibilizar las condiciones en las que se encuentran y atender de forma adecuada sus necesidades específicas (Almeda-Samaranch, 2017). Es preciso seguir analizando sus particularidades para crear programas y acciones que promuevan su rehabilitación, el respeto de sus derechos durante su estancia en prisión, su reinserción social, así como la prevención del delito y de la reincidencia femenina (Espinoza, 2016; AñañosBedriñana \& García-Vita, 2017).

Este proceso se ha venido profundizando a partir de la inclusión de la perspectiva de género, la cual, promueve que se realicen los estudios pertinentes para conocer las experiencias y necesidades de mujeres y hombres en todos los ámbitos, para evaluar las políticas sociales e institucionales existentes y sus efectos, así como, planear y diseñar nuevas iniciativas que favorezcan por igual a ambos géneros y contrarresten la desigualdad y la discriminación (Naciones Unidas, 1997).

La perspectiva de género en el contexto penitenciario tiene la finalidad de conocer $y$ hacer visible el perfil criminológico específico de las mujeres (Yagüe, 2007), y garantizar el respeto de los derechos de las mujeres que se encuentran cumpliendo condena y el reconocimiento de sus necesidades para generar una intervención enfocada en ellas (Cruells et al., 2005).

Los estudios sobre la población reclusa realizados con perspectiva de género revelan importantes cuestiones, entre otras, sobre las condiciones y especificidades de las mujeres privadas de libertad (Acale, 2017; Almeda-Samaranch et al., 2012; AñañosBedriñana \& Yagüe, 2013; Defensor del Pueblo Andaluz, 2006; Holtfreter \& Wattanaporn, 2014), el regreso a las comunidades (Travis et al., 200 I), la discriminación en prisión (Del Val-Cid et al., 20l3), los perfiles adictivos de drogas (Añaños-Bedriñana, 2017a), los tipos de delitos y penas otorgadas (Juanatey, 2018) y la reincidencia (Roth y Zegada, 2016).

Derivado de dichos estudios, un campo emergente y fundamental lo constituyen las relaciones y la influencia de las redes sociales personales de las mujeres para favorecer u obstaculizar los procesos de reinserción (Atkin \& Armstrong, 2018; Berman, 2005; Bui \& Morash, 2010; Clone \& Dehart, 2014; CollicaCox, 2018; Covington, 2003; Cruells et al., 2005; Fernández et al., 2008; García-Vita, 20l7; O’Brien, 200I). En consecuencia, estas relaciones pueden 
constituirse en factores de riesgo mediante las que se involucran en el crimen o, por el contrario, son factores de protección preventivos de delito.

Por todo, el objetivo de este artículo es analizar las investigaciones realizadas en los últimos veinte años sobre las redes personales de las mujeres, identificando los factores de riesgo y protección que inciden en los procesos de reinserción social en las distintas etapas relacionadas con prisión: antes, durante $y$ en semilibertad. El estudio se ejecutó de enero a julio de 2019, las categorías de búsqueda en WOS, Scopus, Google Académico y biblioteca fueron: "redes personales", "apoyo social", "capital social", "relaciones interpersonales" y "relaciones familiares" combinadas con "prisión" y "mujeres" entre los años 1999 y 2019 . Se identificaron 54 estudios virtuales y documentales, 24 procedentes de España y 24 de Estados Unidos, 3 de América Latina (Colombia, México y Argentina), I de la ONU, I de la Unión Europea y I del Reino Unido.

Para llevar a cabo dicho análisis se parte del contexto penitenciario español, en el que la reinserción social y la reeducación son los fines centrales del sistema penitenciario, en cumplimiento del artículo 25.2 de la Constitución Española (1978) y la Ley Orgánica General Penitenciaria en su artículo 59 (SGIP, 1979). Estos objetivos trascienden el acto de la reclusión, brindando a la población reclusa el derecho a una formación integral que le permita contrarrestar las carencias que podría afectarle en la reinserción social, así como desarrollar sus potenciales (Añaños et al., 2019). Un proceso donde personas, grupos e instituciones se involucran en posibilitar las condiciones óptimas de preparación para la libertad y fomentar la participación activa en la sociedad (Villagra, 2008). Esto conlleva que las personas desarrollen la autonomía y las competencias a fin de tomar decisiones que les favorezcan, tanto a sí mismas como a los demás, al igual que poniendo en práctica las habilidades necesarias que les ayuden a desenvolverse eficazmente en los diferentes ámbitos de su vida (Gallizo, 2007).

\section{Teoría de redes sociales y las redes personales}

Las distintas formas de vinculación que establecen los seres humanos dieron lugar al surgimiento de la teoría de redes sociales. La red social se definió en su origen como:
[...] un tercer campo social que no tiene unidades o fronteras, ni una coordinación organizada, los lazos de esta red no son fijos, nuevos lazos se pueden estar formando, mientras que otros se van rompiendo o dejándose en el congelador (Barnes, 1954, p. 43).

Con el paso de los años, la red social se ha convertido en una noción clave y compleja que trasciende las categorías tradicionales que han abordado la interacción social y los límites que imponen la pertenencia a grupos, instituciones, clases sociales, culturas, etc. Actualmente, la categoría de red es una metáfora de gran potencia teóricoepistemológica, explicativa y analítica (Barabási, 2002; Christakis \& Fowler, 2010; Najmanovich, 2007; Wellman, 2000), asimismo cuenta con aplicaciones metodológicas en el campo de la intervención social (Dabas \& Najmanovich, 2002; Fasano, 2010; Machín, 2016; Milanese et al., 2000; Packman, 2002).

Los humanos tienen entre sí diferentes tipos de redes (Molina \& Maya, 2010). Este trabajo aborda las redes egocéntricas, también llamadas personales, cuyo centro es una persona (ego o nodo central) y las relaciones (lazos) que establecen con otras personas -alteri o nodos conectados- (Cachia, 2010). Desde la intervención social, una red personal se define como:

[...] un tejido relacional estable, pero en evolución constituido por nuestros familiares, amigos y conocidos amistosos, conexiones de trabajo y estudio, y relaciones resultantes de nuestra participación en organizaciones comunales formales e informales, incluidas religiosas, sociales, recreativas, políticas, vocacionales, de salud [...] Nuestra red social incluye, de hecho, a todos aquellos con quienes interactuamos y que nos distinguen ( $y$, recíprocamente, distinguimos) de la multitud anónima (Sluzki, 2010, p. 2).

El estudio de las redes personales ha permitido conocer con quiénes se relacionan algunos colectivos específicos, por ejemplo, los/las drogodependientes/ as (Arranz, 2010) y los/las migrantes (Brandes et al., 2010); el tamaño o cantidad de personas involucradas (Fu, 2005); la tipología de intercambios relacionales y la existencia de la reciprocidad (Plickert et al., 2007); los efectos positivos/negativos de las relaciones en su salud (Ávila, 2012; Eckhard, 2018; Visentini et al., 2018), la configuración del apoyo (García-Vita, 2015; Drouhot, 2017), determinar las redes subjetivas de 
amistad (Milanese, 2018) e identificar la dotación del capital social con el que la persona cuenta (Pena \& Sánchez, 2017).

\section{Redes personales de las mujeres en prisión}

Sobre las mujeres privadas de libertad se han realizado investigaciones que muestran la relevancia del análisis de sus redes. En este marco, destacan dos conceptos asociados al objetivo de este estudio: el género y la temporalidad.

En cuanto al género, se identificó una configuración distinta de las redes personales de mujeres y de hombres (De Grande, 2015; Palchykov et al., 2012; Pinker, 2015; Szell \& Thurner, 2013), la que también se refleja en la población reclusa masculina y femenina. Por ejemplo, en prisión, las redes de las internas tienen muy pocos vínculos significativos -padecen soledad y aislamiento- (Fernández et al., 2008) y, en los hombres se mantienen con la misma cantidad de lazos cercanos (Codd, 2008). Otras diferencias donde hay mayor probabilidad de riesgo son: los hombres como parejas/maridos tienden a abandonarlas, no se ocupan adecuadamente de los/as hijos/as o pueden influir en la reincidencia; en cambio las mujeres cuando son parejas/esposas suelen mantenerse al lado apoyando, mayormente asumen la responsabilidad de la crianza de los/as hijos/as y favorecen su reinserción (Codd, 2008; Fernández et al., 2008). También, en los hombres, las relaciones personales (familiares, parejas o amistades) no siempre les llevan a delinquir; en tanto que, en las mujeres pueden propiciar el involucramiento en actos criminales (Covington, 2003; Manjoo, 20I3; O’Brien, 200I).

Respecto a la temporalidad, se observa que las redes personales presentan cambios significativos a través del tiempo. Esto se debe a los diferentes contextos sociales donde se desenvuelven las personas en distintos momentos de su vida, por las etapas de desarrollo o por eventos drásticos inesperados -muerte, accidentes- (Bidart \& Lavenu, 2005; Wrzus et al., 2013) y, en el caso de la población reclusa, el ingreso a prisión (Volker et al., 2016). Al examinar las redes de las mujeres presas se evidencia que sus redes personales no son las mismas antes de prisión, durante prisión y en semilibertad (Bui \& Morash, 2010) y se ha comparado algunos de sus vínculos (madre, padre, pareja, hijos) en cada etapa (Atkin \& Armstrong, 2018). Según Levenrentz (2006) las relaciones sociales de las mujeres que han estado en prisión deben de verse como procesos dinámicos y no estáticos que, al mismo tiempo, son inestables:

[...] una sola relación puede llevar a la mujer a reincidir o a no hacerlo dependiendo del momento en que se analice [...] para que los lazos sociales disuadan a las infractoras, deben ser fuertes, una característica que emerge en el tiempo (Leverentz, 2006, p. 484).

En las ciencias del comportamiento, y concretamente de la criminología, los factores de riesgo son aquellos rasgos de la personalidad o del entorno que incrementan la probabilidad de que se manifieste una conducta antisocial (violencia, delincuencia), no son causales y actúan en conjunto (Farrington et al., 2016). Desde estas disciplinas se han identificado tres clases de factores de riesgo asociados a los problemas de conducta: los individuales, los familiares y los ambientales/contextuales (Justicia et al., 2006; Sanabria \& Uribe, 2010; Webster \& Taylor, 200I). Siguiendo estos autores los factores que se asocian a la red social son: pocas relaciones o aislamiento, pocas habilidades sociales, conflicto familiar agudo, bajo nivel de apoyo emocional, grupos de pares con problemas de conducta antisocial y rechazo a convivir con pares normalizados.

Por su parte, los factores de protección pueden ser de dos tipos, los directos, que son los rasgos de la persona o del entorno que disminuyen la probabilidad de que se manifiesten problemas de conducta $y$, los amortiguadores, que son los que disminuyen la intensidad de los problemas de conducta que puedan presentarse (Durrant, 2017; Lösel \& Farrington, 2012). Los factores de protección que destacan son la familia, las amistades, la escuela y la comunidad de residencia como entornos positivos y seguros; así como algunas características psicológicas de la personalidad como el IQ -inteligencia-, capacidades de autorregulación positiva y actitudes prosociales (Durrant, 2017). La resiliencia es otro factor de protección relevante en el ámbito individual (Lösel \& Farrington, 2012) y, en los procesos socioeducativos relacionales y contextuales (Ruiz et al., 2017).

\section{Etapa 1: redes antes de entrar a prisión}

De acuerdo con O’Brien (200I) los vínculos de las mujeres antes de prisión pueden constituirse en factores de riesgo para los procesos de cumplimiento de condena, de preparación para la libertad y de 
reinserción social. Esto se debe, fundamentalmente, a que las mujeres antes de prisión arrastran las secuelas de una exclusión primaria, caracterizada por múltiples desventajas sociales, culturales, educativas, económicas, etc. (Añaños-Bedriñana, 2012); su infancia y juventud transcurren en entornos familiares y comunitarios inseguros (Melendro, 2017) impregnados de pobreza, violencia y adicción (Dehart, 2008) y con bajas expectativas educativas y laborales formales (Añaños-Bedriñana, 20I3; Yagüe, 2007), de modo que sus vínculos personales se erigen en un conjunto de elementos de riesgo.

\section{Relaciones que implican violencia}

Las mujeres reclusas han padecido algún tipo de violencia (sexual, física y psicológica) durante su infancia, juventud o adultez (Acale, 2017; Covington, 2003; Dehart, 2008; Loinaz, 2016; Yagüe, 2007).

En la Tabla I se observa que, entre el $60 \%$ y $90 \%$ de mujeres en distintos países han sido violentadas. Este abuso ha ocurrido de manera eventual o sistemática en los entornos familiares y domésticos, ocasionado por los padres u otros familiares cercanos y principalmente por la pareja. En estos casos, los vínculos próximos pueden convertirse en factores de riesgo para que las mujeres desde niñas $y$ adolescentes estén expuestas a situaciones de mayor vulnerabilidad social, entre ellas, el consumo de sustancias, la prostitución y las enfermedades mentales (Bui \& Morash, 2010; Dehart, 2008; Yagüe, 2007).

La mayoría de las mujeres reclusas $(57,1 \%$ ) tienen una percepción de su ámbito familiar como negativo y violento en su infancia y juventud, describiendo estas etapas como no agradables y con muchas dificultades, principalmente de maltrato y consumo de drogas (Melendro, 2017). Asimismo, el 60,6\% de las mujeres fueron adictas a drogas antes de entrar a prisión (Añaños-Bedriñana, 2017a), lo cual ha contribuido a una situación y exposición para ser objeto de mayor riesgo.

\section{Relaciones con consecuencias delictivas}

Existe una relación entre los altos porcentajes de violencia/abuso/maltrato y los altos porcentajes de homicidios hacia parejas sentimentales cometidos por las mujeres, después de una sistemática exposición y sometimiento a distintos tipos de violencia (Covington, 2003; Loinaz, 2016; Loinaz \& AndrésPueyo, 2017). En esa línea Dehart (2008) habla de que la polivictimización de las mujeres es un caldo de cultivo para desarrollar carreras criminales. En el $60,7 \%$ de las mujeres en prisión el maltrato se relacionó directamente con el aumento de agresividad hacia la persona agresora (Fontanil et al., 2013).

En otro estudio, el $16,49 \%$ de niños maltratados y el $16,64 \%$ de niñas maltratadas cometieron delitos violentos verificables en su adolescencia y adultez por los que fueron condenados/as a prisión (Topitzes et al., 20I2). Un dato evidenciado en una investigación nacional española con mujeres en prisión, de acuerdo con Añaños-Bedriñana et al. (2019), es que existe una asociación significativa entre las mujeres que siendo menores han tenido medidas judiciales $y$ las que han denunciado maltratos hacia ellas (chi-cuadrado, $P=0,0085$, con una probabilidad de $I, 83$ veces superior).

\section{Tabla I.}

\section{Cifras de violencia y maltrato antes de entrar a prisión}

\section{Mujeres reclusas}

México: $78,7 \%$ revictimización por abuso sexual y el 82,17 \% revictimización por abuso físico. El 72,2\% agresión física o sexual por sus padres antes de los 18 años y el 83,9\% agresión sexual o física por su pareja

España (País Vasco): 67 \% violencia en general, el 33,3\% violencia sexual por su pareja, el $25 \%$ violencia sexual por parte del padre o familiar

España (Oviedo): 7I,4 \% maltrato por alguna persona de su red familiar $y$ el $82,7 \%$ por una o varias parejas

EE. UU.: 90 \% violencia de pareja física o sexual el año anterior al encarcelamiento

EE. UU.: 60 \% violencia sexual, física o psicológica de su esposo/pareja

EE. UU.: 75,49 \% abuso físico y sexual en algún momento de su vida

España (Barcelona): 88,4 \% algún tipo de violencia. En el ámbito

familiar violencia sexual el $59 \%$ y violencia física el $93 \%$

\section{Estudios}

Mejía et al. (2015)

De Miguel (2014)

Fontanil et al. (2013)

Lynch et al. (2012)

Bui \& Morash (2010)

Byrd \& Davis (2009)

Cruells et al. (2005)

Fuente: elaboración propia. 
De acuerdo con el informe de Naciones Unidas (Manjoo, 20l3), una investigación del Departamento de Servicios Correccionales de Nueva York en 2007 reveló que el $67 \%$ de las mujeres que cometieron homicidio asesinó a la persona que había abusado de ellas, y otro estudio mostró que el $66 \%$ de las mujeres actuaron en defensa propia ante personas agresoras cercanas (Gondolf, 1998). Ambos estudios tienen cifras similares a las de Canadá, Australia y Sudáfrica, mientras que en países de Asia la cifra se eleva al $70 \%$.

\section{Relaciones de complicidad y coerción de pareja o familia}

También, existen mujeres que establecen vínculos mediados por la presión o fuerza ejercida para la comisión del acto delictivo (coerción) o por la complicidad.

La coerción se da cuando las mujeres son obligadas o forzadas en su voluntad o conducta, por parte de sus maridos, novios o amigos, llegando hasta la coacción donde hay amenazas o violencia (explícitas/ implícitas), para cometer o ser cómplices de delitos (Codd, 2008; Manjoo, 2013). Estos procesos toman varias formas, por ejemplo, algunas mujeres optan por proteger a parejas/hijos/as, asumiendo la condena sin haber cometido delito o con una participación mínima, ya sea por autosacrificio o por razones económicas (Acale, 2017), o mediadas por la coerción o coacción. Asimismo, hay mujeres que son seducidas/ inducidas por las mafias del narcotráfico a cambio de resolver sus precarias situaciones económicas o, por el contrario, son partícipes con el fin de mejorar su calidad o estilo de vida (Almeda et al., 2012; AñañosBedriñana, 2010).

Otra cuestión, es la influencia del entorno familiar o de la pareja que ha estado o está en prisión, que puede generar un contexto favorable en la concepción del delito e inducir la comisión. Así en el 50,6 \% de las mujeres reclusas en las prisiones españolas se da esta situación, siendo en su mayoría las parejas (52,9 \%) quienes cuentan con este historial, seguida de los/as hermanos/as (8,9 \%) y del padre $(7,4 \%)$ (Melendro, 2017).

\section{Relaciones que apoyan y su ausencia}

Antes de entrar en prisión, según Maidment (2006), menos de la mitad de las mujeres cuenta con relaciones de apoyo familiares; sin embargo, GarcíaVita (2017a) indica en un estudio nacional español que, el $66,7 \%$ recibió apoyo, principalmente de sus madres, el $50 \%$ de sus hermanos y el $47 \%$ de sus hermanas; el $42,2 \%$ de sus padres $y$; en el caso de las amistades, el $52,6 \%$ indica que son amigas y en menor proporción los amigos (46,8\%). Es significativo tener estos datos, ya que dichas relaciones pueden ser un factor de protección en las siguientes etapas: durante prisión y semilibertad.

No obstante, siguiendo a la última autora, habrá que considerar a la otra parte (44,3 \%) que no expresó contar con dichos apoyos familiares y amistosos y, el 6,7 \% manifestó no tener ninguna relación de apoyo en su vida antes de prisión. Por su parte, la ausencia o pérdida de las redes importantes pueden tener un impacto negativo y convertirse en factores de riesgo trascendentales como detonadores de las actividades delictivas, por ejemplo, la muerte de la madre o el padre (Bui \& Morash, 2010).

\section{Etapa 2: redes durante la estancia en prisión}

La prisión es un tipo de institución total "organizado para proteger a la comunidad contra quienes constituyen intencionalmente un peligro para ella, no se propone como finalidad inmediata el bienestar de los reclusos" (Goffman, 200I, p. 18), en el que además los/as reclusos/as se hallan aislados/as durante el tiempo de condena y donde comparten rutinas bajo el control de la administración penitenciaria.

El entorno carcelario se regula con reglas estrictas, horarios y formas de interacción prescritas, se trata de un dispositivo fabricado ex profeso para imponer disciplina (Foucault, 200I). En España la vida en prisión está reglamentada en el denominado Régimen Penitenciario, que agrupa un conjunto de normas o medidas orientadas a la consecución de una convivencia ordenada y pacífica, a fin de permitir alcanzar el ambiente adecuado para el éxito del tratamiento y la retención y custodia de los/as reclusos/as (SGIP, 1996).

"La prisión es de hecho un desarraigo del sujeto de sus condiciones vitales: sociales y subjetivas" (Lagarde, 201I, p. 673). La privación de la libertad impone una fractura en las formas cotidianas de relacionarse, conlleva efectivamente un corte en los lazos que las personas tienen con la sociedad (Bales \& Mears, 2008). Dichas fracturas refieren a los vínculos de pareja, al ejercicio de la maternidad en etapas clave de desarrollo, así como al resto de sus relaciones sociales en el caso de las mujeres, quienes experimentan gran culpabilidad y angustia (Yagüe, 2007). 
A pesar de las circunstancias que impone la vida en prisión, la población reclusa tiene derecho a tener contacto con familiares, pareja, amigos/ as, profesionales que les defiendan y demás personas mediante llamadas telefónicas, cartas y visitas, conforme a lo establecido por las normas internacionales dictadas en las Reglas Nelson Mandela (20I5) y las Reglas de Bangkok (20II) de las Naciones Unidas. Además de ello, de forma inevitable al interior de prisión se establecen relaciones entre las internas y con otras personas. Así, se distinguen dos ámbitos de relación: con personas del exterior y con personas del interior de la prisión (García \& Gutiérrez, 20I2).

Tabla 2.

\section{Red interna y externa en prisión}

\section{Relaciones de las mujeres reclusas al interior de la prisión (red interna)}

La red personal interna está conformada por los vínculos que las mujeres reclusas establecen con las personas con las que conviven diariamente dentro de la prisión. En los centros penitenciarios (CP) solo femeninos la mayoría de sus relaciones son entre mujeres internas y con mujeres del personal penitenciario (Yagüe, 2007), pero si están en prisiones masculinas, aunque haya módulos exclusivos para mujeres, pueden compartir ciertos espacios comunes con los hombres internos (García \& Gutiérrez, 2012), especialmente en el desarrollo de programas de intervención o tratamiento y de acuerdo con una normativa sobre la interacción de ambos.

\begin{tabular}{l|l}
\multicolumn{1}{c|}{ Red interna } & \multicolumn{1}{c}{ Red externa } \\
\hline - Vínculos con mujeres reclusas & - Vínculos con hijos \\
- Vínculos con familiares o pareja en prisión & - Vínculo con pareja o expareja \\
- Vínculos con sus hijos de los 0 a 3 años (SGIP, 1996) & - Vínculos con familiares \\
- Vínculos con hombres reclusos & - Vínculos con amigas y amigos \\
- Vínculos con funcionarias/os & - Vínculos con compañeros de trabajo \\
- Vínculos con profesionales y voluntarios & - Vínculos con mujeres que ya están en \\
- Vínculos con organizaciones y programas & libertad y fueron sus compañeras \\
- asistenciales y servicios sociales & - Vínculos con vecinos y conocidos de familiares y amigos/as \\
\hline laborales y específicos & - Vínculos con organismos, programas, recursos de \\
\hline
\end{tabular}

Fuente: elaboración propia.

Tabla 3.

Factores de riesgo y protección de la red interna

\begin{tabular}{|c|c|}
\hline Factores de riesgo & Factores de protección \\
\hline $\begin{array}{l}\text { Módulos de mujeres en CP masculinos: sin clasificación, } \\
\text { mayor proximidad, mayor conflictividad, limitados } \\
\text { espacios de participación y menos beneficios (García-Vita } \\
\text { \& Melendro, 20I3; Ríos et al., 20I6) } \\
\text { - Traslados frecuentes sin o con cambio de grado } \\
\text { (García \& Gutiérrez, 20I2) } \\
\text { - Relaciones afectivas íntimas entre internas e internos: } \\
\text { embarazos no deseados, abortos, enfermedades, } \\
\text { sexo-servicio y suicidios (García \& Gutiérrez, 20I2) } \\
\text { - Modelo disciplinario punitivo: tensión, desconfianza, conflictos } \\
\text { latentes, quejas por malos tratos, etc. (Fernández-Caballero et } \\
\text { al., 20I2; Pastor \& Huertas, 20I4; Viedma \& Reviriego, 20I2) } \\
\text { - Relaciones de rivalidad, control, sometimiento, } \\
\text { envidia y competitividad entre internas } \\
\text { (García-Vita, 20I7a; Lagarde, 20II) } \\
\text { - Relaciones conflictivas con personal penitenciario } \\
\text { (Viedma \& Reviriego, 20I2) } \\
\text { - Relaciones de indiferencia o desatención con profesionales } \\
\text { (Ríos et al., 20I6) } \\
\text { Calidad de vida deteriorada en el sistema de relaciones } \\
\text { interpersonales al interior del CP } \\
\text { (Almeda, 20I7; Rodríguez et al. 20I8) }\end{array}$ & $\begin{array}{l}\text { - Relaciones de intercambio de favores y servicios } \\
\text { entre internas (García \& Gutiérrez, 20I2) } \\
\text { - Relaciones afectivas íntimas (internas/internos): mayor } \\
\text { atención, apoyo económico, embarazos deseados para trato } \\
\text { diferenciado, etc. (De Miguel, 20I7; García \& Gutiérrez, } \\
20 I 2 \text { ). El 53,I \% de mujeres afirma tener muy buenas } \\
\text { relaciones con los hombres en prisión (García-Vita, 20I7a) } \\
\text { - Relaciones prevalentes de solidaridad, cooperación y } \\
\text { apoyo entre internas (Clone \& Dehart, 20I4; Codd, } \\
2008 ; \text { García-Vita \& Melendro, 20I3; Severance, 2005) } \\
\text { - Régimen disciplinario preventivo: adelantar su cambio } \\
\text { de grado/libertad condicional, becas de estudio, } \\
\text { participación en actividades recreativas/culturales, } \\
\text { atribución de destinos, etc. (Fernández et al., 20I2) } \\
\text { - Relaciones de atención y asesoría con personal penitenciario y } \\
\text { profesionales. El 70 \% de las mujeres en las prisiones españolas } \\
\text { valoró favorablemente las relaciones con los funcionarios o } \\
\text { custodios, profesionales y voluntarios (García-Vita, 20I7a) } \\
\text { Participación en programas socioeducativos y de } \\
\text { formación laboral (Añaños-Bedriñana, 20I7b) }\end{array}$ \\
\hline
\end{tabular}

Fuente: elaboración propia. 
Los autores/as citados en la Tabla 3 señalan que existen factores que pueden perjudicar o ayudar a las mujeres en los procesos de tratamiento o reinserción. Los factores de riesgo hacen referencia a contextos en los que las relaciones pueden carecer de las condiciones necesarias para una sana convivencia, espacios donde impera la inestabilidad, el hacinamiento y la disciplina basada en el castigo, así como las características de las relaciones que generan un ambiente tenso y adverso, como la rivalidad, competitividad, desconfianza, indiferencia, desatención o maltrato, entre otras. Y, por otro lado, los factores de protección pueden ser los programas y las actividades educativas, recreativas y de capacitación, o los espacios comunes en los que la convivencia se regula a partir de los denominados beneficios penitenciarios; así como relaciones que se caracterizan por ser cooperativas, solidarias, de atención, de intercambio y apoyo.

\section{Relaciones de las mujeres reclusas con el exterior de la prisión (red externa)}

Mantener contacto con personas externas a la prisión puede traer importantes beneficios a los/as reclusos/as, entre ellos, la influencia en el éxito de sus procesos de reinserción, así como en la prevención de la reincidencia (Añaños-Bedriñana, 20l7b; Bales \& Mears, 2008; Clone \& Dehart, 2014; Cobbina et al., 2012; Cochran, 20l4; García-Vita, 2017b; King et al., 2005). No obstante, también habrá que considerar los riesgos de algunos tipos de relación y de situaciones que se les presentan a las mujeres para su adaptación durante su estancia en prisión y en su futura reinserción.

Tabla 4.

\section{Factores de protección y riesgo de la red externa}

\begin{tabular}{|c|c|}
\hline Factores de riesgo & Factores de protección \\
\hline $\begin{array}{l}\text { - Ubicación del CP: muy distante, difícil acceso para los } \\
\text { núcleos familiares/amistades. Pocas cárceles solo de mujeres } \\
\text { (4 CP) (Almeda, 20I7; Codd, 2008; Cruells et al., 2005) } \\
\text { - Traslados frecuentes sin o con cambio de } \\
\text { grado (García \& Gutiérrez, 20I2) } \\
\text { - Ruptura o distanciamiento drástico con hijos/as (Bui } \\
\text { \& Morash, 20I0; Codd, 2008; Gust, 20I2) } \\
\text { - Visitas de hijas/os pueden generar comportamientos } \\
\text { violentos, dificultad de adaptación, depresión } \\
\text { o ansiedad (Casey-Acevedo et al., 2004) } \\
\text { - Rompimiento de relaciones: pareja, familiares } \\
\text { (padres), amistades y vecinos (García-Vita, } \\
\text { 20I7b; Fernández et al., 2008; Rope, 20I3) } \\
\text { - Mantener relaciones con parejas/familiares/amistades } \\
\text { con actividades delictivas o consumo de sustancias } \\
\text { (Berg \& Cobbina, 20I7; Coffey \& Elizabeth, 20II) } \\
\text { - Modelo disciplinario punitivo: negación de } \\
\text { permisos/llamadas/visitas y traslados sin cambio } \\
\text { de grado, etc. (Fernández et al., 20I2) } \\
\text { - Ser extranjera dificulta llamadas y visitas de sus } \\
\text { familiares (Castillo \& Ruiz, 20I0; Codd, 2008) } \\
\text { - Ser perfil adicta activa (AA) y adicta en programa } \\
\text { de mantenimiento de metadona (PMM) (Añaños- } \\
\text { Bedriñana, 20I7a; García-Vita, 20I7a) } \\
\text { - Percepción social estigmatizada sobre las reclusas y sanción } \\
\text { social que provoca su abandono (Ayuso, 2003; Codd, 2008) } \\
\text { - Pocas visitas reducen las relaciones y genera desvinculación de } \\
\text { lo que acontece en su medio habitual (Bales \& Mears, 2008) }\end{array}$ & $\begin{array}{l}\text { - Ubicación del CP cerca del lugar de residencia o } \\
\text { el de familiares (García \& Gutiérrez, 20I2) } \\
\text { - Traslado a CP cercano al lugar de residencia familiar o } \\
\text { núcleo de amistades si son extranjeras (Codd, 2008) } \\
\text { - Recuperar o mantener relaciones con familiares/amistades } \\
\text { que dan soporte emocional, cuidados, atención } \\
\text { a sus necesidades, recursos materiales básicos y } \\
\text { apoyo económico (García-Vita, 20I5; 20I7a) } \\
\text { - Ruptura de relaciones con familiares/amistades involucradas } \\
\text { en actividades delictivas y consumo de sustancias } \\
\text { (García-Vita, 20I7a) } \\
\text { - Régimen disciplinario preventivo: permisos de salidas, } \\
\text { aumento en el tiempo o número de llamadas/visitas, salidas } \\
\text { culturales/deportivas, etc. (Fernández et al., 20I2) } \\
\text { - Relaciones significativas y estables con excompañeras } \\
\text { de prisión que dan información y soporte emocional } \\
\text { (Bui \& Morash, 20I0) } \\
\text { - Ser perfil no adicta (NA) o exadicta (EX) } \\
\text { (Añaños-Bedriñana, 20I7a) } \\
\text { - Relaciones duraderas con abogados y personas que } \\
\text { brindan atención legal (García \& Gutiérrez, 20I2) } \\
\text { Contacto frecuente con familiares/amistades } \\
\text { mediante llamadas, visitas y permisos } \\
\text { (Cruells et al., 2005; Meyers et al., 20I7) }\end{array}$ \\
\hline
\end{tabular}

Fuente: elaboración propia. 
De acuerdo con la Tabla 4, los factores de riesgo son los contextos que impiden o limitan a las mujeres poder mantener las relaciones con su red externa, como los CP lejanos a las ciudades - los traslados constantes de un centro a otro; las situaciones particulares como ser extranjera, tener un perfil de adicción AA o PMM; estigmas del entorno que dificultan tener relaciones de apoyo; también, el mantener relación con los/as hijos/as o su ruptura drástica puede repercutir negativamente en el terreno emocional y de adaptación, así como les puede afectar negativamente el rompimiento con la pareja o mantener vínculos con parejas/familiares/ amistades involucrados/as en la droga o delincuencia.

\section{Tabla 5.}

\section{Redes personales de las mujeres en semilibertad}

\begin{tabular}{|c|c|}
\hline Familiares & $\begin{array}{l}\text { - El } 80 \% \text { de mujeres encontró apoyo emocional/material en parientes (padres/ } \\
\text { hermanos/as/abuelos/as)(Bui \& Morash, 20I0). La mayoría encuentra apoyo emocional } \\
\text { en madres e hijos/as (Malek \& Puche, 20I2). El } 55 \% \text { de reincidentes y el } 60 \% \text { de no } \\
\text { reincidentes cuenta con apoyo familiar positivo (Berg \& Cobbina, 20I7) } \\
\text { - Algunas mujeres al regresar a sus comunidades encuentran a sus familiares cercanos involucrados } \\
\text { en drogas, violencia y actividades delictivas (Berg \& Cobbina, 2017; Coffey \& Elizabeth, 20II) }\end{array}$ \\
\hline Pareja & $\begin{array}{l}\text { - Entre el } 33 \text { \% (Leverentz, 2006) y el } 40 \% \text { (Bui \& Morash, 2010) de mujeres inició nuevas relaciones } \\
\text { con exadictos o exreclusos compañeros en grupos, programas de rehabilitación/reinserción, de } \\
\text { quienes recibían apoyo/comprensión y creían que eran mejores relaciones que las anteriores } \\
\text { - El } 57 \% \text { aceptó tener parejas mujeres que iniciaron en prisión y conservan en semilibertad } \\
\text { y estas les aportan apoyo (Leverentz, 2006) } \\
\text { - El } 51 \% \text { (Leverentz, 2006) y el I3,3\% (Bui \& Morash, 2010) evitan tener relaciones de pareja } \\
\text { para prevenir su reincidencia o recaer } \\
\text { - El I2\% mantiene relaciones de pareja con hombres drogodependientes, con actividades delictivas } \\
\text { o en prisión (Leverentz, 2006) } \\
\text { - Las mujeres tienen mayor probabilidad que los hombres de relacionarse nuevamente con } \\
\text { hombres delincuentes y adictos en esta fase y al obtener la libertad (Codd, 2008) }\end{array}$ \\
\hline Hijos/as & $\begin{array}{l}\text { - La mayoría pretende vivir con sus hijos/as a pesar de sentir que les han causado algún daño } \\
\text { (García-Vita, 20I7a) } \\
\text { - Vivir con ellos/as en esta fase y en libertad les genera tensión/estrés. Muchas no se sienten física o } \\
\text { emocionalmente preparadas para atender de sus hijos/as sin ayuda (Codd, 2008; Rope, 20I3) } \\
\text { - Algunas mujeres que han perdido la custodia, tienen la prioridad de recuperarla pero es complicado, por } \\
\text { lo que puede ser un factor de riesgo para reincidencia y recaídas (Leverentz, 2010; Huebner et al., 20I0) }\end{array}$ \\
\hline Amistades & $\begin{array}{l}\text { - El } 80 \text { \% de mujeres dice tener nuevas amistades positivas y que les brindan apoyo (Bui \& Morash, 2010) } \\
\text { - La mayoría intenta romper sus vínculos con conocidos o amigos de antes que están involucrados } \\
\text { en actividades delictivas o drogas (Berg \& Cobbina, 20I7) }\end{array}$ \\
\hline $\begin{array}{l}\text { Excompañeras } \\
\text { de prisión }\end{array}$ & $\begin{array}{l}\text { - La mayoría conserva estas relaciones de amistad, ya que les brindan distintos } \\
\text { tipos de apoyo: emocional, información, hospedaje temporal, contactos laborales, } \\
\text { cuidado de hijos/as (Bui \& Morash, 2010; Severance, 2005; O'Brien, 200I) }\end{array}$ \\
\hline $\begin{array}{l}\text { Compañeras/os de } \\
\text { centros de medio } \\
\text { camino/CIS/grupos } \\
\quad \text { de autoayuda }\end{array}$ & $\begin{array}{l}\text { - En los grupos se aprende un lenguaje común de recuperación que orienta a las mujeres a elegir } \\
\text { con quiénes establecer nuevos vínculos (Leverentz, 2006) } \\
\text { - Las/os compañeras/os de los programas de tratamiento pasan a formar parte de su nueva red } \\
\text { personal y se brindan apoyo mutuo (Bui \& Morash, 2010) } \\
\text { - Se crean relaciones más fuertes en grupos exclusivamente femeninos al compartir experiencias } \\
\text { (Codd, 2008) }\end{array}$ \\
\hline $\begin{array}{l}\text { Personal de } \\
\text { semilibertad/Tercer } \\
\text { grado/Medio abierto }\end{array}$ & $\begin{array}{l}\text { - Estos vínculos les alientan a establecer nuevas relaciones positivas y les desalientan a continuar } \\
\text { relaciones negativas (Leverentz, 20I0) } \\
\text { - El } 75 \% \text { de mujeres consideró buenas y de apoyo sus relaciones con sus supervisores } \\
\text { en esta fase (Bui \& Morash, 2010) } \\
\text { - El estilo de atención y escucha del supervisor genera una relación de apoyo percibida por las reclusas, } \\
\text { pero un estilo autoritario/conformista genera una relación negativa para la reinserción } \\
\text { (Morash et al., 20I8) }\end{array}$ \\
\hline $\begin{array}{l}\text { Voluntarios y } \\
\text { profesionales de } \\
\text { organizaciones civiles e } \\
\text { instituciones religiosas }\end{array}$ & $\begin{array}{l}\text { El } 80 \text { \% de mujeres estableció nuevas relaciones con personas que conocieron en prisión y en esta fase } \\
\text { por las actividades y programas, y el } 55 \% \text { expresó tener buenas relaciones con clérigos y religiosos y } \\
\text { que les fueron de gran ayuda en sus procesos de reinserción (Bui \& Morash, 2010) }\end{array}$ \\
\hline $\begin{array}{l}\text { Compañeras/ } \\
\text { os de trabajo }\end{array}$ & $\begin{array}{l}\text { - El } 65 \% \text { dijo tener nuevas relaciones en sus nuevos empleos y que son relaciones de apoyo } \\
\text { (Bui \& Morash, 2010) }\end{array}$ \\
\hline
\end{tabular}

Fuente: elaboración propia. 
Por su parte, los factores de protección que tendrán repercusiones favorables durante su estancia en prisión son: el estar y mantenerse en CP cercanos a su red externa, gozar de beneficios que les permitan tener mayor contacto con el exterior, romper relaciones antiguas para evitar recaer/reincidir y establecer nuevas relaciones, recuperar o mantener relaciones de apoyo emocional, económico y legal.

\section{Etapa 3: redes en procesos de semilibertad}

La semilibertad es una forma de nombrar a la última fase del cumplimiento de condena que, en España, se conoce como Régimen Abierto, al permitir que las/os internas/os salgan y se desenvuelvan en un medio en el que las medidas de seguridad son flexibles. Se trata de "una mezcla equilibrada entre el internamiento y la libertad vigilada” (Leganés, 2013, p. 176). Es la tercera etapa de la pena privativa de libertad que corresponde al denominado Tercer Grado de la clasificación penitenciaria establecido en el título IV, capítulo II, artículo 100 de la SGIP (1996), siempre y cuando los/as internos/as cumplan el requisito estipulado en el artículo 102.4. La normativa se sitúa en el título III, capítulo III del Reglamento Penitenciario y en su artículo 83 se establece que tiene la finalidad de: "Potenciar las capacidades de inserción social positiva que presentan los penados clasificados en tercer grado, realizando las tareas de apoyo y de asesoramiento y la cooperación necesaria para favorecer su incorporación progresiva al medio social" (SGIP, 1996, p. 37). Por lo tanto, es una fase transitoria en la que los/as internos/as tienen la posibilidad de autogestionar su preparación para la vida en libertad, al mismo tiempo que cuentan con el acompañamiento profesional para ello en un medio abierto.

Los/as reclusos/as al encontrarse en esta fase, que va del encierro total a la reincorporación paulatina al exterior, muestran modificaciones significativas en sus redes personales (Volker et al., 2016).

En el caso de las mujeres en semilibertad, la literatura científica da cuenta de los cambios significativos observados en la conformación de sus redes personales. Por ejemplo, al analizar el tamaño de la red personal en esta fase se encontró que las mujeres con mayor nivel educativo, las que tenían mayores ingresos y las mujeres que se encontraban participando en programas comunitarios de superación personal tenían redes más amplias, de 15 a 20 integrantes en su red. En cambio, las mujeres con bajo nivel educativo, con menores ingresos y las más jóvenes, tenían una red significativamente más pequeña, con menor cantidad de integrantes (Reisig, 2002). Otro cambio observable son los tipos de integrantes de sus redes personales, ya que antes y durante el tiempo de su estancia en prisión eran solo hijos/as, pareja, familiares y amigos/as y, en semilibertad se suman compañeros/as de grupos de autoayuda, excompañeras de prisión, personal penitenciario, profesionales, voluntarios, religiosos/as y compañeros/as de nuevos empleos.

La Tabla 5 refleja dos tendencias, primera, los factores de riesgo señalados aluden a que, parte de las mujeres en el tránsito a la libertad no cuenta con una red segura, puesto que cuando regresan a sus vecindarios/comunidades/barrios están expuestas a situaciones de alto riesgo, al retomar las relaciones con familiares $y$ amistades que siguen delinquiendo o consumiendo drogas. Cabe destacar que existen mujeres que al salir mantienen las relaciones de abuso con parejas previas a prisión y algunas de ellas son parejas que también están o estuvieron en prisión; así también, ocurre que si tienen nuevas parejas vuelven a ser relaciones violentas (Berg \& Cobbina, 2017; Coffey \& Elizabeth, 20I I).

Segunda, los factores de protección que se observan son: redes personales más amplias respecto a la etapa anterior a prisión, más relaciones prosociales y menos relaciones criminales; además, se tiende a evitar la relación romántica o con parejas abusivas o violentas. En esta fase se da una influencia notablemente positiva de parte del personal de prisión, los profesionales y voluntarios de las organizaciones religiosas y civiles (Bui \& Morash, 20l0; Leverentz, 2006).

Uno de los factores clave de protección en semilibertad que influye en el éxito de la reinserción de las mujeres es la conformación y mantenimiento de nuevas redes personales, las cuales dan lugar al acceso de más recursos materiales y emocionales, mejoran la calidad de vida y previenen la reincidencia (Berg \& Huebner, 20II; Bui \& Morash, 2010; Cochran, 20I4; Collica-Cox, 20I8; Maidment, 2006; O'Brien, 200I).

Otra cuestión importante es identificar a las personas prosociales que están presentes en sus redes personales y los tipos de apoyos que les pueden brindar durante su estancia en prisión, pero especialmente cuando están en semilibertad (Wright et al., 2012).

\section{Reflexiones finales}

El presente trabajo evidencia la importancia de las redes personales como elementos esenciales 
de la socialización del ser humano. Los contextos y características de las relaciones que hombres y mujeres establecen con familiares, amistades y otras personas significativas, pueden constituirse, según los casos, en factores de riesgo o protección que inciden en sus comportamientos y situaciones.

El análisis realizado muestra que las redes personales de las mujeres reclusas influyen en el devenir de las historias delictivas que las llevan a prisión, teniendo en cuenta unas condiciones particulares, en función del género, las que se estudian en distintas etapas relacionadas con la prisión: antes de prisión, durante prisión y en semilibertad, como se indica a continuación.

La etapa previa a prisión se caracteriza, por un lado, por una inminente presencia de vínculos de violencia, conflicto o coerción-coacción-complicidad que se establecen en sus entornos más inmediatos, como son la familia y la pareja y, por otro lado, las pocas de relaciones de apoyo o su total ausencia. Las relaciones personales se establecen en contextos prevalentemente permeados por desventajas sociales, educativas, económicas, culturales, etc., en los que se cuenta con limitados recursos para desarrollar trayectorias de vida normalizadas. Con lo cual, se observa mayor presencia de factores de riesgo y menos de protección que pueden incidir en el inicio de las carreras criminales.

Durante la estancia en prisión las redes personales de las mujeres se reestructuran, ya que tratan forzosa e involuntariamente con personas nuevas al interior de prisión, así como se modifica radicalmente el modo en que se relacionan con los miembros de la red externa, además de la limitación de estos contactos. Dentro de la cárcel se recrea un ambiente que puede suscitar dichos factores de riesgo o de protección, dependiendo del modelo de intervención tratamental que se establece y normativiza en cada país y centro, en los que pueden alternar o prevalecer enfoques más punitivos o preventivos de la convivencia.

La conexión con la red externa, pese a las dificultades propias e impuestas por el internamiento, puede favorecer la ruptura de relaciones conflictivas o entornos de riesgos o delictivos $y$, al mismo tiempo recuperar o fortalecer los vínculos emocionales y de apoyo con familiares y amistades. Sin embargo, la ruptura o distanciamiento de sus entornos familiares y sociales, especialmente de los/as hijos/as, influye negativamente en la evolución penitenciaria, al igual que la pervivencia de relaciones con personas que siguen en el mundo delictivo exterior. Existen algunas características como la condición de extranjeras, el uso de drogas y la identificación de reclusa, que dan lugar a la generación de estigmas, agudizando estos distanciamientos o rupturas.

En la fase de semilibertad, las redes personales de las mujeres son diferentes: principalmente en que ellas aumentan y fundan nuevas relaciones; asimismo, se rompen algunas de carácter delictivo o con parejas violentas, consideradas negativas para su reinserción. Los desafíos son enormes al regresar a los entornos de riesgo iniciales. Se trata de un lapso que las pone a prueba en sus tránsitos hacia un cambio de estilo de vida.

En los procesos de la intervención tratamental, durante el periodo de reclusión como en la excarcelación, habrá que considerar que, tanto las familias, las parejas y los entornos comunitarios están presentes e inciden en la reinserción social. Por lo tanto, esto tiene que ver con el desarrollo de los factores de protección personales (resiliencia, autoestima, autoconcepto, autonomía, empoderamiento, etc.) y sociales (educativos, laborales, culturales, relacionales, etc.) que permitan construir relaciones prosociales, con ellas mismas, el entorno y con los profesionales e instituciones que favorecen la reinserción social.

Por último, la revisión sistemática y el desarrollo de las investigaciones que abordan las redes personales de las mujeres permite ver la relevancia de conocer y analizar las mismas, a fin de contar con un estado de la cuestión sólido y fiable que brinde fundamentos para diseñar estrategias de intervención socioeducativas, al tiempo que dé respuestas a los objetivos de la reinserción social y la reeducación, de acuerdo con las características, necesidades e intereses específicos de las personas reclusas, desde una perspectiva de género, buscando mejorar la práctica en el sistema penitenciario y la sociedad en general.

\section{Referencias}

Acale, M. (2017). El género como factor condicionante de la victimización y de la criminalidad femenina. Papers Revista de Sociología, 102(2), 23l-259. https://dialnet.unirioja.es/servlet/ articulo? codigo $=5900025$

Almeda-Samaranch, E. (2017). Criminologías feministas, investigación y cárceles de mujeres en España. Papers Revista de Sociología, 102(2), |5I-|8I. http:// https://dialnet.unirioja.es/ servlet/articulo?codigo $=5900029$

Almeda-Samaranch, E., Di Nella, D., \& Navarro, C. (20I2). Mujeres, cárceles y drogas: datos y reflexiones. Oñati Socio-Legal Series, 2(6), I22145. http://opo.iisj.net/index.php/osls/article/ viewFile//43/38 
Añaños-Bedriñana, F. T. (Coord.). (2010). Las mujeres en las prisiones. La educación social en contextos de riesgo y conflicto. Gedisa.

Añaños-Bedriñana, F. T. (20/2). Violencias y exclusiones en el medio penitenciario. Enfoque socio-educativo y de la paz. Convergencia Revista de Ciencias Sociales, 59, |3-4|.

Añaños-Bedriñana, F. T. (20|3). Formación educativa previa ante las discriminaciones: las mujeres reclusas en España. Revista de Educación, 360, 9I-I I8. https://doi.org//0.4438//988-592XRE-20।3-360-222

Añaños-Bedriñana, F. T. (2017). Prisión. Realidades e intervención socioeducativa y drogodependencias en mujeres. Narcea S. A. de Ediciones.

Añaños-Bedriñana, F. T. (2017). Definición de los perfiles de adicción y rasgos de consumo en mujeres reclusas. En F. T. Añaños-Bedriñana (Dir.), En prisión. Realidades e intervención socioeducativa y drogodependencias en mujeres (pp. 37-55). Narcea S. A. de Ediciones.

Añaños-Bedriñana, F. T., Melendro, M., \& Raya, R. (2019). Mujeres jóvenes con medidas de protección y judiciales y sus tránsitos hacia la prisión. Revista Española de Pedagogía, 77(273), 333-349. https://doi.org/10.22550/ REP77-2-2019-05

Añaños, K. G., Añaños-Bedriñana, F. T., \& Rodríguez, J. A. (2019). Exercising fundamental rights in punitive conditions: education in Spanish prisons. The International Journal of Human Rights, 0(0), I-I5. https://doi.org/10.1080/13 642987.2019.1601084

Arranz, S. (2010). Estrategias para la diversificación de la red personal de personas drogodependientes en proceso de reinserción. Redes-Revista Hispana para el Análisis de Redes Sociales, 18(7), I63-182. http://revista-redes.rediris.es

Atkin-Plunk, C. A., \& Armstrong, G. S. (20/8). Disentangling the relationship between social ties, prison visitation, and recidivism. Criminal Justice and Behavior, 45(10), 1507-1526. https://doi.org/10.1 I77/0093854818772320

Ávila-Toscano, J. H. (Ed.). (20I2). Redes sociales y análisis de redes. Aplicaciones en el contexto comunitario y virtual. Azul Violeta Editores Ltda.

Ayuso, A. (2003). Visión crítica de la reeducación penitenciaria en España. Nau, Libres.

Bales, W. D., \& Mears, D. P. (2008). Inmate social ties and the transition to society does visitation reduce recidivism? Journal of Psychoactive Drugs, 45(3), 287-32I. http://journals.sagepub. com/doi/I0.I I 77/00224278083 I 7574

Barabási, A. L. (2002). Linked. The New Science of Networks. Perseus Publishing.
Barnes, J. A. (1954). Class and committees in a Norwegian Island parish. Human Relations, 7, 39-58.

Berg, M. T., \& Cobbina, J. E. (2017). Cognitive transformation, social ecological settings, and the reentry outcomes of women offenders. Crime \& Delinquency, 63(12), I522-1546. https://doi.org/ I0. I I77/00 I I I 287 I666052 I

Berg, M. T., \& Huebner, B. M. (20II). Reentry and the ties that bind: an examination of social ties, employment, and recidivism. Justice Quarterly, 28(2), 382-4I0. https://doi.org//0.1080/074I 8825.2010 .498383

Berman, J. (2005). Women Offender Transition and Reentry: Gender Responsive Approaches to Transitioning Women Offenders from Prison to the Community. National Institute for Corrections. https://journals.sagepub.com/ doi/pdf/I0.I I 77/00938548I3504406

Bidart, C., \& Lavenu, D. (2005). Evolutions of personal networks and life events. Social Networks, 27(4), 359-376. https://doi.org/10.1016/j. socnet.2004. Il .003

Brandes, U., Lerner, J., Lubbers, M., Mccarty, C., Molina, J. L., \& Nagel, U. (20I0). Recognizing modes of acculturation in personal networks of migrants. Procedia Social and Behavioral Sciences, 4, 4-13. https://doi.org/10.1016/j. sbspro.2010.07.478

Bui, H. N., \& Morash, M. (2010). The impact of network relationships, prison experiences, and internal transformation on women's success after prison release. Journal of Offender Rehabilitation, 49(I), I-22. https:// doi.org/l 0.1080/1050967090343538I

Byrd, P. M., \& Davis, J. L. (2009). Violent behavior in females inmates. Journal of Interpersonal Violence, 24(2), 379-392. https://doi. org/ $10.1177 / 0886260508316475$

Cachia, R. (2010). Las redes personales a la luz del análisis de redes sociales. Redes-Revista Hispana para el Análisis de Redes Sociales, Especial, 7-30.

Casey-Acevedo, K., Bakken, T., \& Karle, A. (2004). Children visiting mothers in prison: the effects in mothers' behaviour and disciplinary adjustment. The Australian and New Zealand Journal of Criminology, 37(I), 4I8-430.

Christakis, N. A., \& Fowler, J. H. (2010). Conectados, el sorprendente poder de las redes sociales y cómo nos afectan. Santillana Ediciones Generales.

Clone, S., \& Dehart, D. (20|4). Social support networks of incarcerated women: types of support, sources of support, and implications for reentry. Journal of Offender Rehabilitation, 53(7), 503-521. https://doi.org//0.1080/1050 9674.2014.944742 
Cobbina, J. E., Huebner, B. M., \& Berg, M. T. (20I2). Men, women, and postrelease offending. Crime \& Delinquency, 58(3), 33I-36I. https:// doi.org/I0.1 I77/00 I I I 287/0382348

Cochran, J. C. (20/4). Breaches in the wall: imprisonment, social support, and recidivism. Journal of Reseach in Crime and Delinquency, 5I(2), 200-229. https://doi. org/I0.II77/00224278/3497963

Codd, H. (2008). In the Shadow of Prison, Families, Imprisonment and Criminal Justice. Willan Publishing.

Coffey-Kellett, N., \& Elizabeth-Willging, C. (20II). Pedagogy of individual choice and female inmate reentry in the $U$. S. Southwest. International Journal of Law and Psychiatry, 34(4), 256-263. https://doi.org/10.1016/j. ijlp.20I I.07.003

Collica-Cox, K. (2018). Female offenders, HIV peer programs, and attachment: the importance of prison-based civilian staff in creating opportunities to cultivate prosocial behaviors. International Journal of Offender Therapy and Comparative Criminology, 62(2), 524-550. https://doi.org/I0.I I77/0306624XI6650680

Covington, S. S. (2003). A woman's journey home. Challenges for female offenders. En J. Travis \& M. Waul (Eds.), Prisoners Once Removed.The impact of Incarceration and Reentry on Children, Families, and Communities. The Urban Institute Press.

Cruells, M., Igareda, N., \& Torrens, M. (2005). Inclusión de la perspectiva de género en las políticas penales, penitenciarias y post-penitenciarias. En M. Cruells \& N. Igareda (Eds.), Mujeres, integración y prisión: un análisis de los procesos de integración sociolaboral de las mujeres presas en Europa (pp. 78-9l). Aurea Editores.

Cruells, M., Torrens, M., \& Igareda, N. (2005). Violencia contra las mujeres. Análisis de la población penitenciaria femenina. http://www. academia.edu/I7859/2/Violencia_contra_ las_mujeres._Análisis_en_el_sistema_ penitenciario

Dabas, E. N., \& Najmanovich, D. (Comp.). (2002). Redes, el lenguaje de los vínculos: hacia la construcción y el fortalecimiento de la sociedad civil. Paidós.

De Grande, P. (20I5). Estructura social y sociabilidad: ¿son desiguales las redes personales? RedesRevista Hispana para el Análisis de Redes Sociales, 26(2), 15-39. https://dialnet.unirioja. es/servlet/articulo?codigo $=5184935$
De Miguel, E. (20I4). Encarcelamiento de mujeres. E castigo penitenciario de la exclusión social y la desigualdad de género. Zerbitzuan, 56, 7586. https://www.zerbitzuan.net/documentos/ zerbitzuan/Encarcelamiento_mujeres castigo_penitenciario_genero.pdf

De Miguel, E. (20I7). Explorando la agencia de las mujeres encarceladas a través de sus experiencias amorosas. Papers Revista de Sociología, I02(2), 3II-335. https://dialnet. unirioja.es/servlet/articulo?codigo $=5900022$

Defensor del Pueblo Andaluz. (2006). Mujeres privadas de libertad en centros penitenciarios deAndalucía. http://www.defensordelpuebloandaluz.es/ sites/default/files/mujeres_presas_0.pdf

Dehart, D. D. (2008). Pathways to prison: impact of victimization in the lives of incarcerated women. Violence Against Women, I4(12), I362-1381. https://doi. org/I0.II77/1077801208327018

Del Val-Cid, C., Viedma, A., \& Frutos, L. (20l3). Sistema de indicadores de discriminación penitenciaria. https://www.researchgate.net/ publication $/ 283716178$

Drouhot, L. G. (2017). Reconsidering "community liberated": how class and the national context shape personal support networks. Social Networks, 48, 57-77. https://doi.org/10.1016/j. socnet.2016.07.005

Durrant, R. (2017). Why do protective factors protect? An evolutionary developmental perspective. Aggression and Violent Behavior, 32, 4-10. https://doi.org/10.1016/j. avb.2016.12.002

Eckhard, J. (2018). Indicators of social isolation: a comparison based on survey data from Germany. Social Indicators Research, 139(3), 963-988. https://doi.org//0.1007/s I I205$017-174 \mid-y$

Espinoza, O. (20I6). Mujeres privadas de libertad. ¿Es posible su reinserción social? Caderno $\mathrm{CRH}$, 29(3), 93-106. https://doi.org//0.1590/S010349792016000400007

Farrington, D. P., Ttofi, M. M., \& Piquero, A. R. (20I6). Risk, promotive, and protective factors in youth offending: results from the Cambridge study in delinquent development. Journal of Criminal Justice, 45, 63-70. https://doi. org/l0.1016/j.jcrimjus.2016.02.014

Fasano, L. (20I0). Tejiendo redes: el papel de las redes sociales en la salud y el bienestar. Gran Aldea Editores.

Fernández, A., García del Moral, N., Urbano, A. \& Arostegui, E. (2008). Prisión y género. Efectos del encarcelamiento en mujeres y hombres presos y en su entorno familiar. Asociación Zubiko. 
Fernández-Caballero, M., Del Hierro, E.. \& Archilla, M. (20/2). "Mediación penitenciaria": una nueva prupuesta para mediar en una cárcel de mujeres. Revista de Mediación, 10, 39-43. https:// revistademediacion.com/wp-content/up loads/20 I3/08/Revista-Mediacion- I I-07l.pdf

Fontanil, Y., Alcedo, M. A., Fernández, R., \& Ezama, E. (20/3). Mujeres en prisión: un estudio sobre la prevalencia del maltrato. RES Revista Española de Sociología, 20, 21-37. http://www. fes-sociologia.com/files/res/20/03.pdf

Foucault, M. (2002). Vigilar y castigar. Siglo Veintiuno Editores.

Fu, Y. (2005). Measuring personal networks with daily contacts: a single-item survey question and the contact diary. Social Networks, 27, 169-186.

Gallizo, M. (2007). Reinserción social de drogodependientes ingresados en centros penitenciarios. Salud y Drogas, 7(I), 57-74.

García-Vita, M. del M. (2016). Redes de apoyo y entornos sociofamiliares en mujeres reclusas: análisis de las relaciones con las drogas, el acompañamiento en prisión y los procesos hacia la reinserción social. Universidad de Granada. https://dialnet. unirioja.es/servlet/tesis?codigo $=5635 \mathrm{I}$

García-Vita, M. del M. (20I7a). ¿Qué sucede con la familia y los vínculos sociales de las mujeres cuando son condenadas a prisión? En F. T. Añaños-Bedriñana (Dir.), En prisión. Realidades e intervención socioeducativa $y$ drogodependencias en mujeres (pp. 121-133). Narcea S. A. de Ediciones.

García-Vita, M. del M. (20I7b). Análisis de los apoyos y conflictos sociofamiliares de las mujeres en prisiones españolas. Revista de Paz y Conflictos, 10, I89-2II. http://revistaseug.ugr.es/index. php/revpaz/article/view/522I

García-Vita, M. del M. \& Melendro, M. (20/3). El ambiente en prisión: la atención recibida por las reclusas y las relaciones intramuros. Pedagogía Social. Revista Interuniversitaria, 22, 43-56. https://doi.org/ I0.7I 79/PSRI

García de Cortázar, M. \& Gutiérrez, J. (20I2). Relaciones en prisión. En C. Del Val y A. Viedma (Eds.), Condenadas a la desigualdad. Sistema de indicadores de discriminación penitenciaria (PP. 143-I72). Icaria.

Goffman, E. (200I). Internados: ensayos sobre la situación social de los enfermos mentales. Amorrortu.

Gust, L. V. (20/2). Inside the beltway: can policy reduce the collateral damage caused by the criminal justice system? American Journal of Orthopsychiatry, 82(2), I74-180. https://doi. org/I0.IIII/j.1939-0025.20I2.0II56.x

Holtfreter, K., \& Wattanaporn, K. A. (20I4). The transition from prison to community initiative: an examination of gender responsiveness for female offender reentry. Criminal Justice and Behavior, 4I(I), 4I-57. https://doi. org/l 0.1 I 77/00938548/3504406

Huebner, B. M., Dejong, C., \& Cobbina, J. (2010). Women coming home: long-term patterns of recidivism. Justice Quarterly, 27(2), 225-254. https://doi.org/I0.1080/074/8820902870486

Juanatey, C. (2018). Delincuencia y población penitenciaria femeninas: situación actual de las mujeres en prisión en España. Revista Electrónica de Ciencia Penal y Criminología, 20, I -32. http://criminet.ugr.es/recpc

Justicia, F., Benítez, J. L., Pichardo, M. C., Fernández, E., García, T., \& Fernández, M. (2006). Aproximación a un nuevo modelo explicativo del comportamiento antisocial. Electronic Journal of Research in Educational Psychology, 4(2), | 3 |- | 50. https://doi.org/ | 0.25 | | 5/ejrep. v4i9. 1187

King, R. S., Mauer, M., \& Young, M. C. (2005). Incarceration and Crime: A Complex Relationship. The Sentencing Project. https:// www.sentencingproject.org/wp-content/ uploads/20 I 6/0 I/Incarceration-and-CrimeA-Complex-Relationship.pdf

Lagarde, M. (20II). Los cautiverios de las mujeres. Romanya Valls.

Leganés, S. (2013). Clasificación penitenciaria y medio abierto. Universidad de Valencia, Facultad de Derecho. http://roderic.uv.es/ handle/ I0550/26359

Leverentz, A. (2010). People, places, and things: how female ex-prisoners negotiate their neighborhood context. Journal of Contemporary Ethnography, 39(6), 646-68I. https://doi.org/10.1 I77/089/241610377787

Leverentz, A. M. (2006). The love of a good man? Romantic relationships as a source of support or hidrance for female exoffenders. Journal of Research in Crime and Delinquency, 43(4), 459-488. https://doi. org/l 0.1 I 77/0022427806293323

Loinaz, I. (2016). Cuando "el" delincuente es "ella": intervención con mujeres violentas. Anuario de Psicologia Jurídica, 26(I), 4I-50. https://doi. org/l0.1016/j.apj.2016.04.006

Lösel, F., \& Farrington, D. P. (20I2). Direct protective and buffering protective factors in the development of youth violence. American Journal of Preventive Medicine, 43(2), S8-S23. https://doi.org/ I0.10 16/j.amepre.2012.04.029

Lynch, S. M., Fritch, A., \& Heath, N. M. (20I2). Looking beneath the surface: the nature of incarcerated women's experiences of interpersonal violence, treatment needs, and mental health. Feminist Criminology, 7(4), 38I-400. https:// doi.org/I0.I I77//557085 I I 2439224 
Machín, J. (2016). Meta-modelo ECO2 : una introducción a sus fundamentos matemáticos. Fundación Río Arronte. https://www.researchgate. net/publication/30I780I0I_Metamodelo_ECO2_Una_introduccion_a_sus_ fundamentos matematicos

Maidment, M. (2006). Doing Time in the Outside: Deconstructing the Benevolent Community. University of Toronto Press.

Malek, S. \& Puche, I., (20I2). Experiencia de intervención con mujeres privadas de libertad en etapa de pre-egreso. Revista Electrónica de Psicología Social "Poiésis" FUNLAM, 24, I-II. http://www.funlam.edu.co/revistas/index. $\mathrm{php} /$ poiesis/article/view/516

Manjoo, R. (2013). Causas, condiciones y consecuencias de la encarcelación para las mujeres. Naciones Unidas. https://undocs.org/es/A/68/340

Mejía, B., Zea, P., Romero, M., \& Saldívar, G. (20I5). Traumatic experiences and re-victimization of female inmates undergoing treatment for substance abuse. Substance Abuse Treatment, Prevention, and Policy, I0(5), 4-II. https:// substanceabusepolicy.biomedcentral.com/ track/pdf/I0.I I86/I747-597X-10-5

Melendro, M. (2017). La infancia y la juventud de las mujeres presas, una referencia clave para la intervención socio. En F. T. Añaños-Bedriñana (Dir.), En prisión. Realidades e intervención socioeducativa y drogodependencias en mujeres (pp. 89-103). Narcea S. A. de Ediciones.

Meyers, T. J., Wright, K. A., Young, J. T. N., \& Tasca, M. (2017). Social support from outside the walls: examining the role of relationship dynamics among inmates and visitors. Journal of Criminal Justice, 52(May), 57-67. https://doi. org/10.1016/j.jcrimjus.2017.07.012

Milanese, E. (20I8). Características estructurales de las redes subjetivas, estudio exploratorio. I-I8.

Molina, J. L., \& Maya, I. (Eds.). (20I0). Elementos para el trabajo en red. Apuntes desde el análisis de redes sociales. Redes-Revista Hispana para el Análisis de Redes Sociales, Especial, I-II6. http://revista-redes. rediris. es/webredes/novedades/Trabajoenred_ materialeseducativos.pdf

Morash, M., Kashy, D. A., Smith, S. W., \& Cobbina, J. E. (20l8). Is the nature of communication relevant to the supportiveness of women's relationships with probation and parole agents? International Journal of OffenderTherapy and Comparative Criminology, 62(6), I629- 1647. https://doi.org/I0.I I77/0306624X 6689832

Naciones Unidas \& Asamblea General de la UNODC. (1997). Informe del Consejo Económico y Social correspondiente a 1997. Vol. (A/52/3/Re). https://digitallibrary.un.org/record/27|316/ files/A_52_3_Rev.I-ES.pdf

Najmanovich, D. (2007). El desafío de la complejidad: redes, cartografías dinámicas y mundos implicados. Revista Internacional de Filosofía Iberoamericana y Teoría Social, 38, 7I-82. www. redalyc.org/pdf/279/27903806.pdf

'Brien, P. (200I). Making it in the "Free World": Women in Transition from Prison. University of New York Press.

Packman, M. (2002). Redes: metáfora para práctica de intervención social. En E. Dabas \& D. Najmanovich (Eds.). Redes. El lenguaje de los vínculos (pp. 294-302). Paidós.

Palchykov, V., Kaski, K., Kertész, J., Barabási, A. L. ,\& Dunbar, R. I. M. (20I2). Sex differences in intimate relationships. Scientific Reports, 2(370), I-5.https://doi.org/10.1038/srep00370

Pastor, E.. \& Huertas, E. (20|4). Mediación penitenciaria, una alternativa a la resolución pacífica de conflictos entre internos. Pedagogía Social. Revista Interuniversitaria, 2(23), 199-229. https://doi.org// 0.7/79/PSRI

Pena-López, J. A., \& Sánchez-Santos, J. M. (2017). Individual social capital: accessibility and mobilization of resources embedded in social networks. Social Networks, 49, I-II. https:// doi.org/10.1016/j.socnet.2016.11.003

Pinker, S. (20I5). The Village Effect, Why Face to Face Contact Matters. Atlantic Books.

Plickert, G., Rochelle, R. C., \& Wellman, B. (2007). It's not who you know, it's how you know them: Who exchanges what with whom? Social Networks, 29, 405-429. https://doi. org/l0.1016/j.socnet.2007.01.007

Reisig, M. D., Holtfreter, K., \& Morash, M. (2002). Social capital among women offenders. Journal of Contemporary Criminal Justice, /8(2), I67-187. https://doi.org/ I0.| I77/I043986202018002004

Ríos, J., Pascual, E., Sevogia, J. L., Etxebarria, X., \& Lozano, F. (2016). Mediación penal, penitenciaria y encuentros restaurativos. Experiencias para reducir el sufrimiento en el sistema penal. Universidad Pontificia Comillas.

Rodríguez, J., Larrauri, E., \& Güerri, C. (2018). Percepción de la calidad de vida en prisión. La importancia de una buena organización y un trato digno. Revista Internacional de Sociología, 76(2), I-20. https://doi.org/10.3989/ris.2018. 76.2.16.159

Rope, O. (2013). Filling the research gap: women prisoners and their families. En Justice for Children of Prisoners (Ed.), Looking Forward: Impacts, Successes and Obstacles (pp. 20-2I). EUROCHIPS. 
Roth, E., \& Zegada, A. (2016). La mujer frente al delito: factores asociados a la reincidencia delictiva femenina. Ajayu, 14(I), 102-1 20.

Ruiz-Román, C., Calderón-Almendros, I., \& Juárez, J. (20I7). La resiliencia como forma de resistir a la exclusión social: un análisis comparativo de casos. Pedagogía Social. Revista Interuniversitaria, 29, I29-|4I. https://doi.org/I0.7I79/PSRI

Sanabria, A. M., \& Uribe, A. F. (20/0). Factores psicosociales de riesgo asociados a conductas problemáticas en jóvenes infractores y no infractores. Diversitas: Perspectivas en Psicología, 6(2), 257-274. https://doi.org//0.15332/ sl794-9998.2010.0002.04

Severance, T. A. (2005). "You know who you can go to": cooperation and exchange between incarcerated women. The Prison Journal, 85(3), 343-367. https://doi. org/ I 0.1 I 77/0032885505279522

SGIP. (1996). Reglamento penitenciario. https:// boe.es/buscar/pdf/I 996/BOE-A- 1996-3307consolidado.pdf.

Sluzki, C. E. (20I0). Redes sociales personales y salud: implicaciones conceptuales y clínicas de su impacto recíproco. Family Systems and Health, 28(I), I-I8. https://sluzki.com/publications/ articles// 29/personal-social-networks-andhealth-conceptual-and-clinical-implicationsof-their-reciprocal-impact

Szell, M., \& Thurner, S. (20I3). How women organize social networks different from men. Scientific Reports, I, 20-22. https://doi.org//0.1038/ srep01214

Topitzes, J., Mersky, J. P., \& Reynolds, A. J. (20I2). From child maltreatment to violent offending: an examination of mixed -gender and genderspecific models. Journal of Interpersonal Violence, 27(I2), 2322-2347. https://doi. org/I0.। I77/08862605 I|4335 I0

Travis, J., Solomon, A. L., \& Waul, M. (200I). From Prison to Home. The Dimensions and Consequences of Prisoner Reentry. T. U. Institute. ttp://citeseerx. ist.psu.edu/viewdoc/load;jsessionid= ODEA 3 F 9 E 289 A 94 D 88 D B 83273 $3853 \mathrm{D} 5 \mathrm{DB}$ ? do $i=|0.1 .1 .2| 2.6235 \& \mathrm{rep}=$ rep I \&type $=$ pdf

Viedma, A. \& Reviriego, F. (20I2). Ejecución penal y punitividad. La convivencia cotidiana con el castigo. En C. Del Val \& A. Viedma (Eds.), Condenadas a la desigualdad. Sistema de indicadores de discriminación penitenciaria (pp. |73-2|4). Icaria.
Villagra, C. (2008). Reinserción: lecciones para una política pública. Debates Penitenciarios, 6, 3-I8. https://www.cesc.uchile.cl/publica ciones/debates_penitenciarios_06.pdf

Visentini, C., Cassidy, M., Bird, V. J., \& Priebe, S. (2018). Social networks of patients with chronic depression: a systematic review. Journal of Affective Disorders, 24 I (June), 57I-578. https://doi.org/10.1016/j.jad.2018.08.022

Volker, B., De Cuyper, R., Mollenhorst, G., Dirkzwager, A., Van Der Laan., P. \& Nieuwbeerta, P. (2016). Changes in the social networks of prisoners: a comparison of their networks before and after imprisonment. Social Networks, 47, 47-58. https://doi. org/10.1016/j.socnet.2016.04.004

Webster-Stratton, C., \& Taylor, T. K. (200I). Nipping early risk factors in the bud: preventing substance abuse, delinquency, and violence in adolescence through interventions targeted at young children (0-8 Years). Prevention Science, 2(3), 165-192. https://doi.org//0. I023/A:I0II510923900

Wellman, B. (2000). El análisis estructural: del método y la metáfora a la teoría y la sustancia. Política y Sociedad, 33, I I-40.

Wright, E. M., DeHart, D. D., Koons-Witt, B. A., \& Crittenden, C. A. (20I2). "Buffers" against crime? Exploring the roles and limitations of positive relationships among women in prison. Punishment and Society, I5(I), 7I-95. https://doi.org/I0.I I 77//4624745 I 2466199

Wrzus, C., Hänel, M., Wagner, J., \& Neyer, F. J. (20/3). Social network changes and life events across the life span: a meta-analysis. Psychological Bulletin, I39(I), 53-80. https://doi.org/ I0.1037/a002860 I

Yagüe, C. (2007). Mujeres en prisión. Intervención basada en sus características, necesidades y demandas. Revista Española de Investigación Criminológica: REIC, 4(5), I-24. https://www.corteidh.or.cr/tablas/r39686.pdf 